\title{
Effect of Single and Two Step Application of Antioxidant Incorporated Bleaching Agents on Bond Strength of Resin Composite and Surface Changes in Enamel
}

\author{
Megha Nair, Ravi Nesamani, Kavitha Sanjeev*, Mahalaxmi Sekar and Senthil Renganathan
}

SRM Dental College Ramapuram, Chennai, Tamil Nadu, India

*Corresponding author: Kavitha Sanjeev, Professor, Department of Conservative Dentistry and Endodontics, SRM Dental College Ramapuram, Chennai, Tamil Nadu, India, Tel: +251911091969; E-mail: kavithasanjeev02@gmail.com

Received date: August 30, 2016; Accepted date: October 04, 2016; Published date: October 11, 2016

Copyright: @ 2016 Nair M, et al. This is an open-access article distributed under the terms of the Creative Commons Attribution License, which permits unrestricted use, distribution and reproduction in any medium, provided the original author and source are credited.

\begin{abstract}
Objective: The aim of this in vitro study was to incorporate two antioxidants namely sweet potato and grape seed extracts with $30 \%$ hydrogen peroxide and to evaluate its effect on the enamel surface and the bond strength of resin composite to enamel.

Materials and Methods: Labial enamel surfaces of 90 extracted human maxillary central incisors were randomly divided into 6 groups of 15 each based on different bleaching protocols. Groups I and II were allotted to negative (no bleaching) and positive (bleaching with 30\% hydrogen peroxide only) controls. The rest were the experimental groups; bleaching with $30 \%$ hydrogen peroxide incorporated with $2 \%$ sweet potato and $5 \%$ grape seed extracts (Groups III and IV respectively) and bleaching followed by application of sweet potato and grape seed extracts (Groups V and VI respectively). Following bleaching procedure, 5 specimens were evaluated for surface changes under SEM and the remaining 10 specimens were immediately restored with resin composite and were subjected to shear bond strength evaluation under universal testing machine.
\end{abstract}

Results: Significantly higher shear bond strength values were observed in teeth treated with the experimental bleaching solution containing $2 \%$ sweet potato extract (Group III) as compared to the other groups. Comparatively lesser morphological changes in enamel were also observed under SEM for Group III. 5\% grape seed extract showed better results when used as a separate step rather than when incorporated in the bleaching agent.

Conclusion: Addition of $2 \%$ sweet potato extract in bleaching agent reduces deleterious effects on enamel, enhances bonding of resin composites to bleached enamel significantly and does not require additional clinical steps.

Keywords: Tooth enamel; Bleaching; Proanthocyanidine; Sweet potato; Antioxidant

\section{Introduction}

With an increase in the demand for aesthetics, vital bleaching has become a popular conservative treatment modality for discolored teeth as it preserves tooth structure and avoids invasive restorative interventions. Current vital and non-vital bleaching techniques employ oxidizing agents such as hydrogen peroxide and other peroxide releasing agents [1].

Hydrogen peroxide undergoes ionic dissociation to form free radicals such as nascent oxygen, hydroxyl radical, per-hydroxyl and superoxide anions when they are applied to enamel [2]. These free radicals are highly reactive and break down larger chromogenic pigments into smaller, less intensive ones [2-4]. Though bleaching agent comes in varying concentrations ranging from $10-38 \%$ and in different formulations, hydrogen peroxide remains the primary ingredient. The amount of oxygen release differs based on the concentration of hydrogen peroxide. Although effective bleaching is achieved in terms of aesthetics, the deleterious effect on enamel in any form/concentration still remains the same. Various studies have demonstrated weakening of enamel surface due to oxidation of organic and inorganic components and dissolution of enamel matrix leading to loss of mineral content due to bleaching. Zalkind et al. revealed that bleaching with peroxides over a short period of time caused Nano morphological alteration of the enamel surface whereas prolonged exposure resulted in micro morphological changes such as surface pitting, crater and gap formation [5].

Moreover the presence of this residual peroxide decreases the ability of resin adhesives to penetrate enamel for micro-mechanical retention and inhibits polymerization needed for clinical success [6]. The most common method used to counteract this problem was to perform all restorative procedures following bleaching after a delay of 2-3 weeks [2]. However, several recent studies have proposed the use of antioxidants namely sodium ascorbate, proanthocyanidin and lycopene to counteract the effects of free radicals following bleaching procedure thus enabling restorative procedure to be performed immediately $[2,6]$. These naturally occurring antioxidants have shown to have free radical scavenging ability which can reverse the reduced bond strength of resin to bleached enamel. However, so far, the antioxidants have been used as a separate application procedure following bleaching. Though the bond strength of the resins can be reversed, the destruction of enamel surface caused by the bleaching 
Citation: Nair M, Nesamani R, Sanjeev K, Sekar M, Renganathan S (2016) Effect of Single and Two Step Application of Antioxidant Incorporated Bleaching Agents on Bond Strength of Resin Composite and Surface Changes in Enamel. Biol Med (Aligarh) 8: 348. doi: $10.4172 / 0974-8369.1000348$

Page 2 of 5

cannot be reversed. Moreover, this two-step procedure results in extended clinical time overall $[2,6,7]$.

Studies done in the field of food chemistry have indicated that phytochemical present in tubers possess antioxidant and free radical scavenging activity. Sweet potato (Ipomoea batatas $L$ ) is one such plant tuber, which has a high content of enzymes such as polyphenol peroxidase (PO), catalase (CAT), and superoxide dismutase (SOD) [8]. Sarath et al. formulated a bleaching agent containing vegetative enzyme extract (sweet potato) obtained from plant tubers. They concluded that the addition of sweet potato extract to two different concentrations of hydrogen peroxide not only resulted in the restoration of natural tooth color but also decreased the effects of bleaching on the enamel morphology, compared to the use of hydrogen peroxide alone [9]. However the bond strength of resin composites to enamel surface was not evaluated. Hence, in this study sweet potato containing vegetative enzymes was incorporated with the purest and higher concentration of hydrogen peroxide, considering the fact that, the other ingredients like thickening and stabilizing agent in the bleaching solution are inactive and do not influence the release of oxygen free radical [10].

The aim of this study was to comparatively evaluate their effects on the enamel surface and the bond strength of composite resin to enamel. The null hypothesis was that incorporation of these extracts will not have any effect.

\section{Materials and Methods}

\section{Preparation of experimental bleaching solutions}

200 gms of sweet potato (purple color) were cleaned, cut into cubes and blended in a blender. Debris and fibers from the potato concentrate were filtered out. The potato concentrate was then transferred into a cooling centrifuge to be processed at $2000 \mathrm{rpm}$ for about 20 minutes under $4^{\circ} \mathrm{C}$. The clear liquid thus obtained was $2 \%$ sweet potato extract (SPE). It was then refrigerated at $4^{\circ} \mathrm{C}$ until use. 1 $\mathrm{ml}$ of SPE was blended with $28 \mathrm{ml}$ of PBS (Phosphate Buffer Saline 100 $\mathrm{mM}$ ) and $1 \mathrm{ml}$ of $30 \%$ hydrogen peroxide (Thermo Fisher Scientific India Pvt Ltd, Mumbai, India) to prepare $30 \mathrm{ml}$ of the experimental bleaching solution (BSP).

Similarly, 5 grams of grape seed extract in the form of powder (Puritans Pride INC Oakdale, NY, USA) was collected from the capsules and dissolved in $100 \mathrm{ml}$ of distilled water to make 5\% (GSE). $1 \mathrm{ml}$ of GSE was blended with $28 \mathrm{ml}$ of PBS and $1 \mathrm{ml}$ of $30 \% \mathrm{H} 2 \mathrm{O} 2$ to prepare $30 \mathrm{ml}$ of experimental bleaching solution (BGS).

\section{Specimen preparation}

90 freshly extracted caries free, human maxillary central incisor teeth were collected, and the crowns were embedded in self-cure acrylic resin block till cemento-enamel junction, keeping only the coronal portion exposed. Labial surfaces were flattened with 600 grit silicon carbide paper (Moyco Precision Abrasives, Montgomeryville, PA, USA). The fifteen teeth served as negative control (Group I) and did not receive any bleaching treatment. The labial enamel surfaces of 75 specimens (Group II, III, IV, V, VI; n=15 each) were subjected to bleaching protocol based on the type of bleaching solutions and number of steps involved. Group II - Bleached with 30\% $\mathrm{H} 2 \mathrm{O} 2$ only. Group-III and IV were allotted to the single application of the experimental bleaching solutions BSPand BGS respectively.

The two step procedures of bleaching with $30 \% \mathrm{H} 2 \mathrm{O} 2$ followed by rinsing and application of 2\% SPE and 5\% GSE for 10 minutes respectively constituted Group V and VI.

Scanning electron microscopic analysis: To evaluate the effect of bleaching solutions on the surface morphology of enamel, five specimens were subjected to scanning electron microscopic analysis (SEM JEOL model, JSE-5610 LV). The specimens were vacuum desiccated first in alcohol and subsequently in acetone, followed by sputter-coating with gold. Micrographs were taken at a magnification of 1000x. The entire exposed labial surface was scanned and the most critical areas were selected for SEM photomicrographs.

Shear bond strength evaluation: In the remaining 10 samples, the labial enamel surfaces of each group were etched with $37 \%$ phosphoric acid (Total Etch etching gel, Ivoclar Vivadent, Schaan, Liechtenstein) for 15 seconds using micro brush, rinsed with distilled water for 20s, bonded with Adper Single Bond (3M ESPE, Dental Products, St Paul, MN, USA) and cured for 20s. This was followed by composite build up (Filtek Z350, 3M ESPE, Dental Products) using Teflon mold of $3 \mathrm{~mm}$ diameter and $5 \mathrm{~mm}$ height. All the specimens were stored in distilled water for 24 hours before shear bond strength testing.

Each specimen was loaded in Universal Testing Machine (UTM) (LR 100K, Lloyd Instruments, Largo, FL, USA) in such a way that the long axis of the specimens was parallel to the direction of the applied forces. The knife-edge was loaded at the interface between the composite and the enamel surface. The shear bond strength (SBS) was measured in shear mode at a crosshead speed of $0.5 \mathrm{~mm} / \mathrm{min}$ until fracture occurred. Data were tabulated and statistically analyzed. Kruskal-Wallis one-way analysis of variance (ANOVA) and post-hoc Tukey's test was used to calculate the level of significance.

\section{Results}

The results of this study are given in Table 1 . SEM images of all the groups are shown in Figure 1(a-f). Graphic representation of the comparison of mean shear bond strength between groups is given in Figures 2 and 3.

\begin{tabular}{|l|l|l|l|}
\hline \multicolumn{1}{|c|}{ Groups } & \multicolumn{1}{|c|}{$\begin{array}{c}\text { Mean } \pm \text { SD } \\
\text { Mpa }\end{array}$} & Overall p-value & \multicolumn{1}{|c|}{ Significant } \\
\hline I & $15.6 \pm 5.18$ & & III, V Vs I, II \\
\cline { 1 - 2 } II & $8.53 \pm 4.43$ & & IV, VI Vs I,II \\
\hline III & $21.14 \pm 10.05$ & \multirow{2}{*}{$<0.004$ (Sig) } & III Vs I, II, IV, V, VI \\
\hline IV & $17.82 \pm 5.73$ & & \\
\hline V & $13.75 \pm 4.8$ & & \\
\hline VI & $19.14 \pm 8.2$ & & \\
\hline
\end{tabular}

Table 1: Comparison of mean shear bond strength of different study groups. 
Citation: Nair M, Nesamani R, Sanjeev K, Sekar M, Renganathan S (2016) Effect of Single and Two Step Application of Antioxidant Incorporated Bleaching Agents on Bond Strength of Resin Composite and Surface Changes in Enamel. Biol Med (Aligarh) 8: 348. doi: $10.4172 / 0974-8369.1000348$

Page 3 of 5
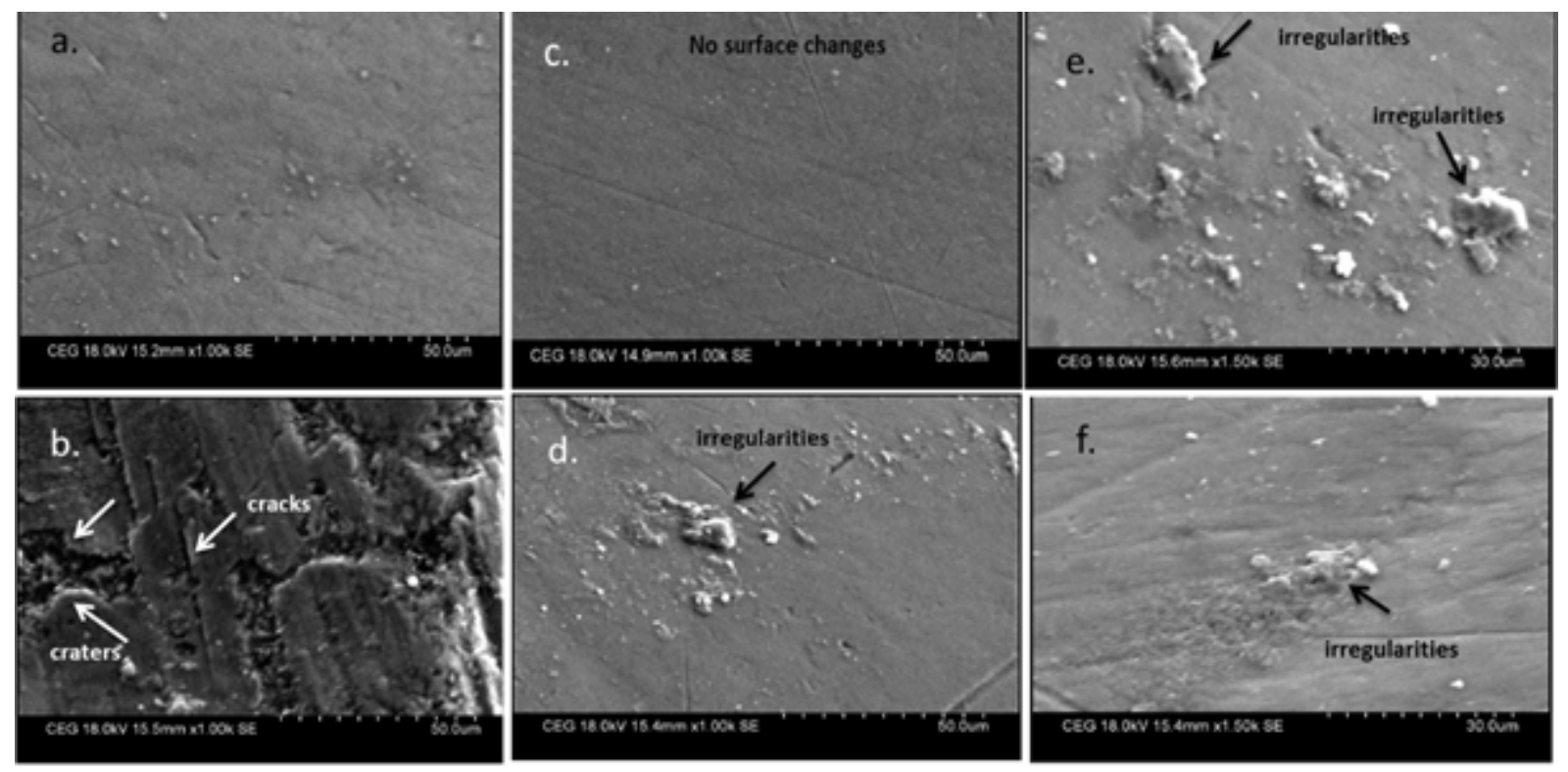

Figure 1: (a) SEM micrograph of Group-I (No bleaching). No surface changes; (b) SEM micrograph of Group-II ( $30 \% \mathrm{H}_{2} \mathrm{O}_{2}$ ); Presence of more number of cracks and craters; (c) SEM micrograph of Group-III (BSP). Absence of irregularities, craters or cracks; (d) SEM micrograph of Group-IV (BGS). Presence of irregularities; (e) SEM micrograph of Group-V (B/SP). More number of irregularities and cracks; (f) SEM micrograph of Group-VI (B/GS). Lesser number of irregularities, with absence of cracks.

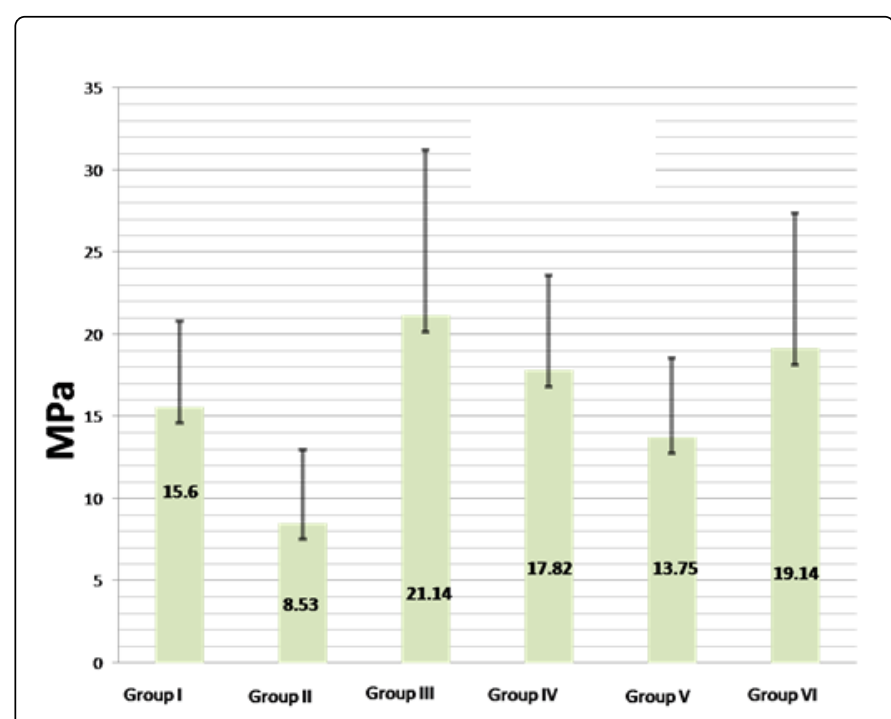

Figure 2: Graphical representation of the comparison of mean bond strength between different study groups.

The mean SBS of positive control was significantly lower than negative control where no bleaching was done. When comparing all the groups, Group-III (BSP) showed significantly higher SBS values $(21.14 \pm 10.05)$ than Group VI (B/GS; $19.14 \pm 8.2)$, Group V (B/ SP(13.75 \pm 4.8 ), Group IV (BGS; $17.82 \pm 5.73$ ) and the control groups.

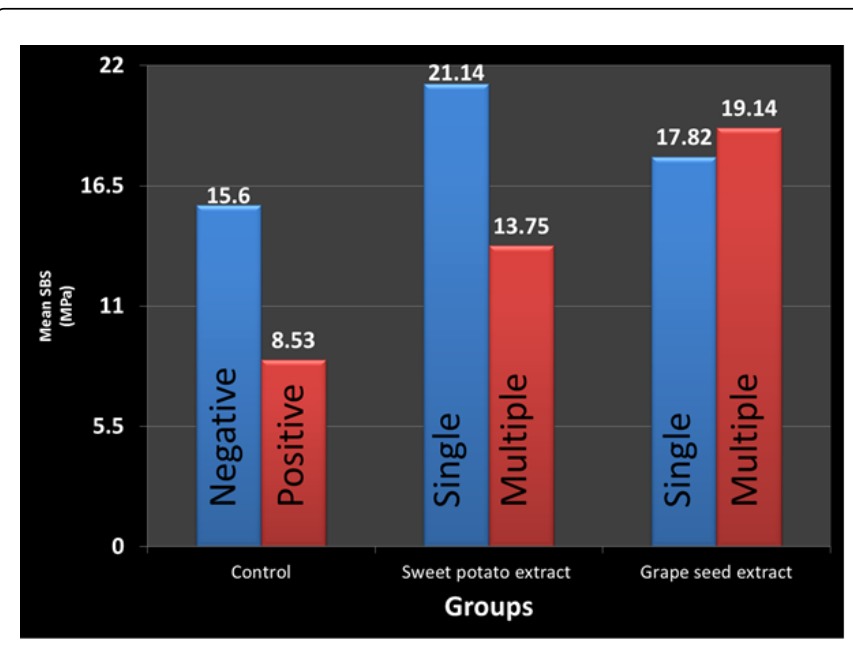

Figure 3: Graphical representation showing the mean bond strength values of controls; $2 \%$ sweet potato extract [single and multiple step - Group III and V] and 5\% grape seed extract [single and multiple step - Group IV and VI].

When comparing the single step procedure, Group III showed significantly higher SBS values than Group IV. Among the two-step groups, Group VI showed significantly higher SBS values than Group V.

Both the antioxidant groups, either when used in single step or two steps, showed significantly higher bond strength than the positive control (Group-II; bleaching with $30 \% \mathrm{H}_{2} \mathrm{O}_{2}$ only). Among the 
Citation: Nair M, Nesamani R, Sanjeev K, Sekar M, Renganathan S (2016) Effect of Single and Two Step Application of Antioxidant Incorporated Bleaching Agents on Bond Strength of Resin Composite and Surface Changes in Enamel. Biol Med (Aligarh) 8: 348. doi: $10.4172 / 0974-8369.1000348$

Page 4 of 5

antioxidants, single step procedure with $2 \%$ SPE showed significantly higher SBS values compared to the other experimental groups.

SEM micrographs of Group II $-30 \% \mathrm{H}_{2} \mathrm{O}_{2}$ (Figure $1 \mathrm{~b}$ ) showed a roughened enamel surface. Presence of cracks and craters was also evident. SEM micrographs of Group I (Figure 1a) and Group III (Figure 1c) showed absence of surface irregularities, cracks or craters. Group V (Figure 1e) showed presence of more morphological surface irregularities compared Group IV and VI (Figures 1d and 1f).

\section{Discussion}

Vital tooth bleaching procedures are the most commonly opted conservative and effective treatment options to treat discolored teeth $[6,11]$. Various oxidizing agents such as hydrogen peroxide and carbamide peroxide readily decompose with the release of free radicals when it encounters substances with which it can react.

Though bleaching is the most sought after aesthetic treatment, in the present era of aesthetic dentistry, several shortcomings are faced by the operator/dentist, the most challenging problem lies in the waiting period after bleaching before any adhesive restoration can be done.

The poor bonding surface following bleaching occurs due to the retained peroxide in the subsurface layer of enamel which interferes with the bonding of the resin composite [12]. And also the alterations in enamel structure, as a result of increased porosity as manifested by loss of prismatic form and an over etched appearance leads to lower bond strength of resin composite [13]. Several studies have been suggested to treat the bleached surface with alcohol, organic solvents and proposed removal of superficial layer of enamel before restoration, the general approach was to postpone the bonding procedure from 24 hours to 4 weeks $[6,14]$. To overcome this delay, Vidhya et al. have shown that application of proanthocyanidin as a separate step post bleaching resulted in reversal of bond strength of bleached enamel immediately [2]. Proanthocyanidins are high molecular weight polymers that comprise monomeric flavan-3-ol, $(+)$ catechin and $(-)$ epicatechin [2].

In 2013, Sarath et al. [9] showed that use of 35\% and 10\% hydrogen peroxide containing sweet potato extract as bleaching agents not only resulted in better bleach, but also decreased the effects of bleaching on the enamel morphology, compared to the use of hydrogen peroxide alone, the effects being more pronounced in higher concentration of $\mathrm{H}_{2} \mathrm{O}_{2}[8,15,16]$. This eliminated the separate step of application of antioxidant.

This present study was undertaken to evaluate the effect of this phytochemical on the immediate bond strength of composite resin to bleached enamel and compare with a known antioxidant, proanthocyanidin in both single and two applications protocol.

According to the results of our study, among the control groups, the mean SBS of resin composite to bleached enamel, the positive control $(8.53 \pm 4.43 \mathrm{MPa})$ was significantly lower than the negative unbleached control $(15.6 \pm 5.18 \mathrm{MPa})$. This was evident in the SEM images, in which Group II showed presence of cracks and roughed surface morphology (Figure 1b) compared to Group I (Figure 1a). This is in accordance with the previous studies done by Andre et al. who observed greater resin tag length for unbleached enamel compared to bleached enamel [17].

The mean bond strength of all 4 experimental groups were significantly higher when compared to the control groups.
Of the SPE groups, Group-III $(21.14 \pm 10.05 \mathrm{MPa})$ yielded significantly higher bond strength compared to Group-V $(13.75 \pm 4.8$ $\mathrm{MPa})$. The higher efficacy of the combined solution can be attributed to the enzymatic action of the catalase present in the sweet potato extract, which, when added to hydrogen peroxide, reduces its high activation energy thereby increasing the rate of release of free radicals [9]. Activation energy is critical to any intended chemical reaction. In order to initiate the reaction, this minimum energy barrier has to be overcome by the reactant molecules, so that the reaction can be activated, whereby, subsequent reaction products can be produced. Certain enzymes, when added to the reactant molecules can reduce the level of activation energy and increase the efficacy or rate of the chemical reaction [9].

When SPE is added to hydrogen peroxide in specific concentrations, the enzyme catalase present in sweet potato extract combines temporarily with $\mathrm{H}_{2} \mathrm{O}_{2}$ molecule forming a catalase- hydrogen peroxide complex. Catalase $\left(\mathrm{H}_{2} \mathrm{O}_{2}\right.$ oxidoreductase $)$ a tetramer, constitutes four polypeptide chains, each over 500 amino acids long. It comprises four (iron) groups that enables the enzyme to react with the hydrogen peroxide [18].

This combination places added stress on the intermolecular bonds holding the substrate molecules together, thereby lowering its activation energy. The activation energy of $\mathrm{H}_{2} \mathrm{O}_{2}$ is thus reduced from $75 \mathrm{KJ} / \mathrm{mol}$ to $21 \mathrm{KJ} / \mathrm{mol}$. Owing to this, large amount of free radicals is released within a short period of time. This in turn reduces the time the bleaching agent is in contact with the bleached tooth, thus lessening the possibility of creating grooves or micro pores across the surface of the tooth.

Sweet potato contains plurality of antioxidant molecules, which are enzymatic or non- enzymatic in nature, such as superoxide dismutase (SOD), anthocyanin, tocopherol and beta-carotene. They exert high scavenging action $[8,19-22]$. But their efficacy to quench completely may not have been possible, because the enzyme catalase, apart from speeding up the reaction, it also decomposes hydrogen peroxide $\left(\mathrm{H}_{2} \mathrm{O}_{2}\right)$ into oxygen and water. This is achieving by two different modes of enzymatic activity: the catalytic mode of activity $\left(2 \mathrm{H}_{2} \mathrm{O}_{2} \rightarrow\right.$ $\left.\mathrm{O}_{2}+2 \mathrm{H}_{2} \mathrm{O}\right)$ and the peroxidase mode of activity $\left(\mathrm{H}_{2} \mathrm{O}_{2}+\mathrm{AH}_{2} \rightarrow \mathrm{A}\right.$ $+2 \mathrm{H}_{2} \mathrm{O}$ ). One molecule of catalase can convert millions of molecules of hydrogen peroxide to water and oxygen, per second. Thus, there must have been an outburst of ample amount of oxygen free radicals to fraction the molecular chains of pigments that caused discoloration. This is confirmed by the SEM micrographs (Figure 1c), which showed the presence of minimal irregularities compared to Group-1 (due to presence of oxygen radicals), but, lesser morphological changes compared to other groups (due to antioxidant potential of the extract) $[9,18]$. Our results are in concurrence with the findings of the study done by Sarath et al. [9].

Presence of more number of irregularities in SEM images and lesser shear bond strength of Group-V (sweet potato as a separate step) compared to Group III, confirms the insufficient amount of antioxidants to quench completely, due to additional release of oxygen radical separately by enzyme catalyze.

Proanthocyanidin, a high molecular weight polymer which comprises of catechin is found in high concentrations in natural sources such as grape seed extract, pine bark extract, cranberries, lemon tree bark and hazelnut tree leaves. Although many studies have shown the efficacy of proanthocyanidin in the reversal of bond strength, there are no studies so far to evaluate its efficacy when 
Citation: Nair M, Nesamani R, Sanjeev K, Sekar M, Renganathan S (2016) Effect of Single and Two Step Application of Antioxidant Incorporated Bleaching Agents on Bond Strength of Resin Composite and Surface Changes in Enamel. Biol Med (Aligarh) 8: 348. doi: $10.4172 / 0974-8369.1000348$

Page 5 of 5

combined with bleaching agent. In the present in-vitro study GroupIV (BGS; $17.82 \pm 5.73 \mathrm{MPa})$ and Group-VI (B/GS; $19.14 \pm 8.2 \mathrm{MPa})$ showed an increase in shear bond strength when compared to Group II (control; $8.53 \pm 4.43 \mathrm{MPa}$ ) in which no antioxidants was added. This proved that the deleterious effect of $30 \%$ hydrogen peroxide was probably reduced due to the specificity of proanthocyanidins for hydroxyl free radicals, the presence of multiple donor sites that trap superoxide radicals, and the esterification of epicatechin by gallic acid in proanthocyanidins, which enhances the free radical scavenging ability $[2,16]$.

Among the two experimental groups of GSE, Group-IV (BGS) showed lesser bond strength than Group-VI (B/GS), this could have been due to the lesser ratio of the antioxidant activity of the proanthocyanidin to the free radical released, which could have resulting in incomplete quenching of the free radicals released by hydrogen peroxide. SEM micrographs of the present study. Group IV and $\mathrm{V}$ (Figure 1d and e) showed presence of more morphological surface irregularities compared Group VI (Figure 1f).

But when comparing the SPE groups with GSE groups, the shear bond strength of both the GSE groups were lesser, though not statistically significant. The higher bond strength of SPE compared to GSE could be due to the presence of proanthocyanidin in the skin of the sweet potato in addition to the other flavonoids present within, and its capacity to lower the activation energy of hydrogen peroxide. Thus this synergetic activity could be responsible for the additional scavenging potential when compared to proanthocyanidin alone and its combination [23]. This is evident in the SEM micrographs of the present study. Group III (Figure 1c) showed absence of surface irregularities compared Group IV and VI (Figure 1d and f).

\section{Conclusion}

The addition of $2 \%$ sweet potato extract to the $30 \%$ hydrogen peroxide bleaching agent reduces clinical steps and the deleterious effect on enamel surface. It also enhances the bonding of composite resins to bleached enamel. Hence this experimental bleaching solution containing $2 \%$ sweet potato extract seems to be very promising and can be considered for routine clinical application in the management of discolored teeth. The efficacy of 5\% GSE is better appreciated when used as a separate step following bleaching.

\section{Conflict of Interest}

The authors have no financial interest in any of the companies or products mentioned in the article.

\section{References}

1. Chen HP, Chang CH, Liu JK, Chuang SF, Yang JY (2008) Effect of fluoride containing bleaching agents on enamel surface properties. Journal of Dentistry 36: 718-725.

2. Vidhya S, Srinivasulu S, Sujatha M, Mahalaxmi S (2011) Effect of grape seed extract on the bond strength of bleached enamel. Operative Dentistry 36: 433-438.

3. Torres CRG, Koga AF, Borges AB (2006) The effects of anti-oxidant agents as neutralizers of bleaching agents on enamel bond strength. Braz J Oral Sci 16: 971-976.

4. Garcia-Godoy F, Dodge WW, Donohue M, O'Quinn JA (1993) Composite resin bond strength after enamel bleaching. Operative Dentistry 18: 144-147.
5. Zalkind M, Arwaz JR, Goldman A, Rotstein I (1996) Surface morphology changes in human enamel, dentin and cementum following bleaching: a scanning electron microscopy study. Endod Dent Traumatol 12: 82-88.

6. Turkun M, Celik EU, Kaya AD, Arici M (2009) Can the hydrogel form of sodium ascorbate be used to reverse compromised bond strength after bleaching? The Journal of Adhesive Dentistry 11: 35-40.

7. Bacci ACL, Florio FM, Basting RT (2007) Effect of a 37\% carbamide peroxide bleaching agent activated by halogen light or light emiting diodes (LEDs) on enamel surface roughness microhardness. Revista de Odontologia Da UNESP 36: 351-355.

8. Teow CC, Truong VD, McFeeters RF, Thompson RL, Pecota KV, et al. (2007) Antioxidant activities, phenolic and $\beta$-carotene contents of sweet potato genotypes with varying flesh colours. Food Chemistry 103: 829-838.

9. Sarath G, James V, Karthikeyan K, Vidhya S, Kavitha S, et al. (2013) Effect of bleaching with two different concentrations of hydrogen peroxide containing sweet potato extract as an additive on human enamel: An in vitro spectrophotometric and scanning electron microscopy analysis. Journal of Conservative Dentistry 16: 45.

10. Ourique SA, Arrais CA, Cassoni A, Ota-Tsuzuki C, Rodrigues JA (2011) Effect of different concentrations of carbamide peroxide and bleaching periods on the roughness of dental ceramics. Braz oral res 25: 453-8.

11. Joiner A (2007) Review of the effects of peroxide on enamel and dentine properties. Journal of Dentistry 35: 889-896.

12. Aslihan U, Aykent F (2003) Bond Strengths of Porcelain Laminate Veneers to Tooth Surfaces Prepared with Acid and Er, Cr: YSGG Laser Etching. The Journal of Prosthetic Dentistry 90: 24-30.

13. Josey Al, Meyers IA, Romaniuk K, Symons Al (1996) The Effect of a Vital Bleaching Technique on Enamel Surface Morphology and the Bonding of Composite Resin to Enamel. Journal of Oral Rehabilitation 23: 244-50.

14. Soobrattee MA, Neergheen VS, Luximon-Ramma A, Aruoma OI, Bahorun T (2005) Phenolics as potential antioxidant therapeutic agents: mechanism and actions. Mutation Research 579: 200-213.

15. Heim KE, Tagliaferro AR, Bobilya DJ (2002) Flavonoid antioxidants: chemistry, metabolism and structure-activity relationships. The Journal of Nutritional Biochemistry 13: 572-584.

16. Chedea VS, Braicu C, Socaciu C (2010) Antioxidant/prooxidant Activity of a Polyphenolic Grape Seed Extract. Food Chemistry 121: 132-39.

17. Andrew J (2006) The Bleaching of Teeth: A Review of the Literature. Journal of Dentistry 34: 412-19.

18. Kabel AM (2014) Free Radicals and Antioxidants: Role of Enzymes and Nutrition. World Journal of Nutrition and Health 2: 35-38.

19. Truong VD, McFeeters RF, Thompson RT, Dean LL, Shofran B (2007) Phenolic acid content and composition in leaves and roots of common commercial sweet potato (Ipomea batatas L.) cultivars in the United States. J Food Sci 72: C343-349.

20. van den Berg H, Faulks R, Granado HF, Hirschberg J, Olmedilla B, et al. (2000) The potential for the improvement of carotenoid levels in foods and the likely systemic effects. Journal of the Science of Food and Agriculture 80: 880-912.

21. Ghasemzadeh A, Talei D, Jaafar HZE, Juraimi AS, Mohamed MTM, et al. (2016) Plant-growth regulators alter phytochemical constituents and pharmaceutical quality in Sweet potato (Ipomoea batatas L.). BMC Complementary and Alternative Medicine 16: 152.

22. Padda MS, Picha DH (2008) Quantification of phenolic acids and antioxidant activity in sweetpotato genotypes. Scientia Horticulturae 119: $17-20$.

23. Huang Q, Chen C, Peng H (2009) Study on Extraction of Proanthocyanidins from Sweet Potato Skin [J]. Acta Agriculturae Jiangxi 7: 43 . 